\title{
Detection of Whole Blood Glucose Concentration by Fourier-Transform Raman Spectroscopy and Artificial Neural Networks
}

\author{
Wang Qiaoyun ${ }^{1^{\star}}$, Wu Guangfei ${ }^{2}$, Shan Peng ${ }^{1}$, Li Zhigang ${ }^{1}$, Hu Sheng ${ }^{1}$, \\ Ma Zhenhe ${ }^{1}$ \\ ${ }^{1}$ College of Information Science and Engineering, Northeastern University, Shenyang, China \\ ${ }^{2}$ Department of endocrinology, the First Hospital in Qinhuangdao, Qinhuangdao, China
}

\begin{abstract}
Objectives: Diabetes, which is caused by the disorder of blood glucose, has been one of the most important metabolic diseases worldwide. In order to avoid the diabetes-related complications, such as blindness and loss of limbs, the diet adjustment and insulin therapy are need to check glucose level at least four to five times a day. The aim of this paper was to investigate the potential of Fourier-Transform Raman spectroscopy as a diagnostic tool to monitor the blood glucose, triglycerides and total cholesterol levels. Methods: Raman spectroscopy were acquired from 116 individuals with diabetes patients, patients and healthy patients in order to gain an insight into the determination of biochemical changes for the diabetes diagnose. The human whole blood was examined at 1064nm excitation laser source. Results: A new method with modified sample selection algorithm named (K-Means) KM is developed and applied to the Raman spectroscopy of whole blood. The algorithms have been successfully applied to improve the prediction accuracy. This method can detect the glucose concentration in the human whole blood without complicated sample preparation procedures. Conclusions: The experimental results show that the Raman spectroscopy technology has enormous clinical potential as a rapid diagnostic tool for diabetes diseases.
\end{abstract}

Keywords: Whole blood, Glucose, Raman spectroscopy, Artificial neural network, Diabetes diseases

\section{Introduction}

Diabetes is one of the largest global health emergencies of the $21^{\text {st }}$ century. The number of diabetes world wide are currently estimated to 415 million, and to reach 642 million by 2040, 1 in 11 adults have diabetes. By 2040, 1 adult in 10 will have diabetes, 1 in 7 births is affected by gestational diabetes, every 6 seconds a person dies from diabetes[1]. Diabetes Mellitus is a metabolic disorder and characterized by the high blood glucose levels which depend on the secretion of insulin from the pancreas. Diabetic patients rely on fingerprick methods (2-4 times daily) method to control blood glucose level, which measures a small sample of blood, usually drawn from the tip of the finger. It is painful and has risk of spreading infectious diseases. The need for a portable handheld easy and safely handled at home, for monitoring blood glucose in individuals every now and then becomes pertinent.

For the last decades a goal has been to develop a noninvasive method for determination of BG. As this will ease the life of diabetics significantly. The research has nearly exclusively focused on near infrared (NIR)measurement in the subcutaneous tissue[2]. Due to the strong water absorption affecting and the short penetration depth, the NIR is suit to analyze the dried blood. Raman spectroscopy has been recognized as a potentially useful method in physics, chemistry and biology fields for investigations of vibrational modes. In the past few years, the use of Raman spectroscopy to identify human body fluids, important components in many crime scene investigations, has been proposed and demonstrated[3]. Lowering costs, minimal sample and almost no reagent are needed for Raman spectroscopy.

The output spectra at the chosen wavelengths are provided to an artificial neural network (ANN) which is used to analyze the relationship between the Raman spectra and the glucose levels. ANNs are used to solve variety of problems like pattern recognition, prediction, and optimization[4-7]. Most of 
the existing models of neural networks interpret biological behaviour in terms of time averaging techniques rather than directly emulating them. On the other hand, the inverse delayed function model is a universal neuron model including the Bonhoeffer Van der Pol model similar to the biological nerve cell.

The aim of this study was to investigate the possibilities of using FT-Raman spectroscopic measurements to detect and quantify blood glucose concentration. The nonlinearity of the system has been suitably linearized using ANN. The results show that this method is robust, accurate and repeatable for fast blood glucose analysis.

\section{Experimental setup}

The Raman spectra were measured with a Bruker MultiRAM (Bremen, Germany) Fourier Transform (FT) Raman spectrometer, as shown in Fig.1. The excitation light was generated by a near infrared Nd:YAG laser at $1064 \mathrm{~nm}$. The laser power incident on the samples was 100mw. The low power laser was used to ensure that there is no thermal degration of the sample during analysis, nor will be a problem to be used for the analysis of biological tissue. A high sensitivity Ge detector that was cooled with liquid nitrogen was used to measure the back scattered radiation. The Raman spectral data were collected using an OPUS 7.0 (Bruker Optics, Germany). Each sample was scanned in the quartz cuvette 3 times at room temperature. All of the Raman spectra represented an average of 512 scans over the $4000-400 \mathrm{~cm}-1$ range (Stokes shift) at a $6 \mathrm{~cm}-1$ spectral resolution and a laser power of $500 \mathrm{~mW}$. A full factorial experimental design was used to determine the most appropriate acquisition parameters, such as the number of accumulations, the acquisition time and the laser power.

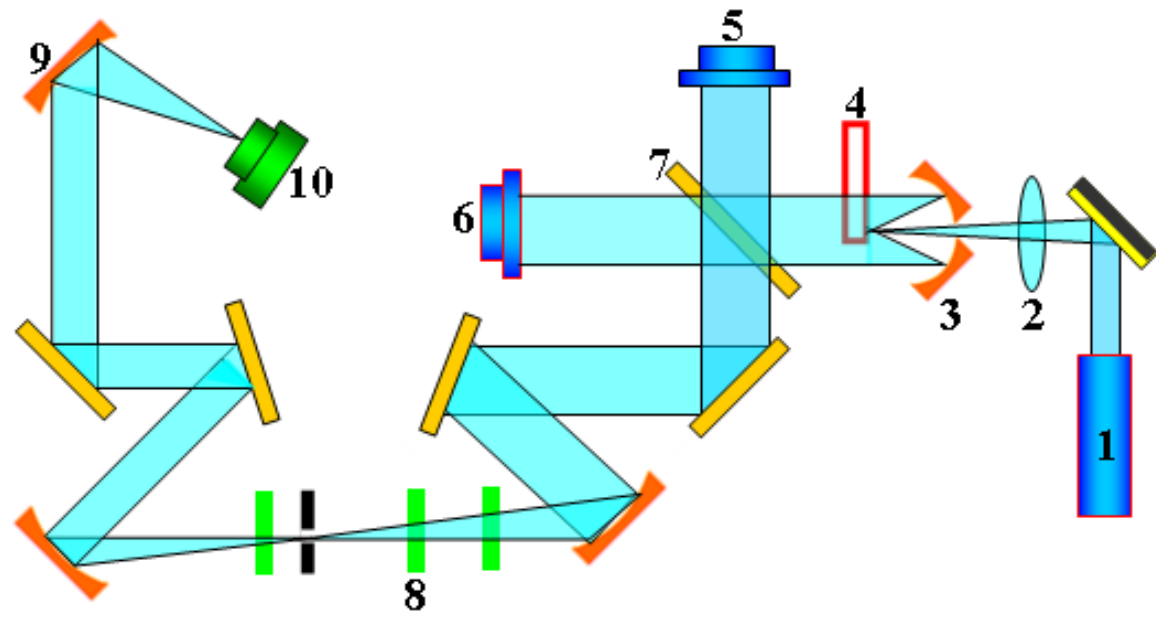

Figure 1. The schematic of the Raman spectroscopy. 1. 1064nm near infrared Nd:YAG laser, 2, lens, 3. paraboloid converging lens, 4. sample, 5. fixed mirror, 6. moving mirror,7. beamsplitter, 8. light filter, 9. Condensing lens, 10. Ge detector cooled by liquid nitrogen

A quartz cuvette $(25 \mathrm{~mL})$ with optically flat faces, a mirrored backside and near infrared (IR) transparency was used as the cell for the liquid samples. Chromium oxide was flame-sealed into the cuvette and used as a standard to correct for the effects on the Raman intensity from the variations in the laser power. This procedure allowed for the mixed Raman spectrum of the chromium oxide standard and the sample to be collected synchronously without any extra optical configuration. The top of cuvette was sealed with a Teflon plastic cap to prevent the liquid from evaporating.

Each sample measurement was repeated three times, and the measurement with the largest deviation with respect to the average was eliminated. To eliminate the effects of the test environment, all of the experiments were performed at room temperature $\left(25^{\circ} \mathrm{C}\right)$ with the humidity at ambient level in the laboratory. Before acquiring the Raman spectra, the quartz cuvette was warmed from room temperature to $36.6{ }^{\circ} \mathrm{C}$ and left at $36.6{ }^{\circ} \mathrm{C}$ for 1 hour. 
The $5 \mathrm{~mL}$ blood was collected by venipuncture from healthy donors into Vacutainer blood collection tubes containing Ethylenediaminetetraacetic acid (EDTA) as an anticoagulant. Blood samples were stored in a $4{ }^{\circ} \mathrm{C}$ refrigerator immediately after collection until required for measurements or additional separation steps.

Clinical reference methods

Measurements by reference methods were carried out at the first hospital in Qinhuangdao with reagents supplied by the manufacturer. Glucose was measured by a hexokinase method[8, 9] (Catalog nr. 04404483 190, CV\%<1.5\%). All procedures recommended by the manufacturer were followed.

\section{Data Processing}

Due to the interference between the glucose and other substance in the blood, there is a nonlinear relationship between the glucose concentration and the Raman spectra. To compensate for this nonlinearity, the ANN is ideal model for approximating such functions[4, 10]. Initially, the back propagation artificial neural network comprising of two layers is chosen. The hidden neurons in hidden layer is 8, which is decided by the number of latent variable in PLS, and the outputs has only one neuron. In the hidden layer, the delayed weighted sum of inputs with the inverse tan-sigmoid activation functions are processed. The transfer function of output layers was purelin function. The system has been validated with the data of 50 patients. The R, mean square error (MSE) and RMSEP are used to choose the model.

\section{Results}

The original whole blood Raman spectra of 116 samples recommended by the OPUS software are shown in Fig.2.

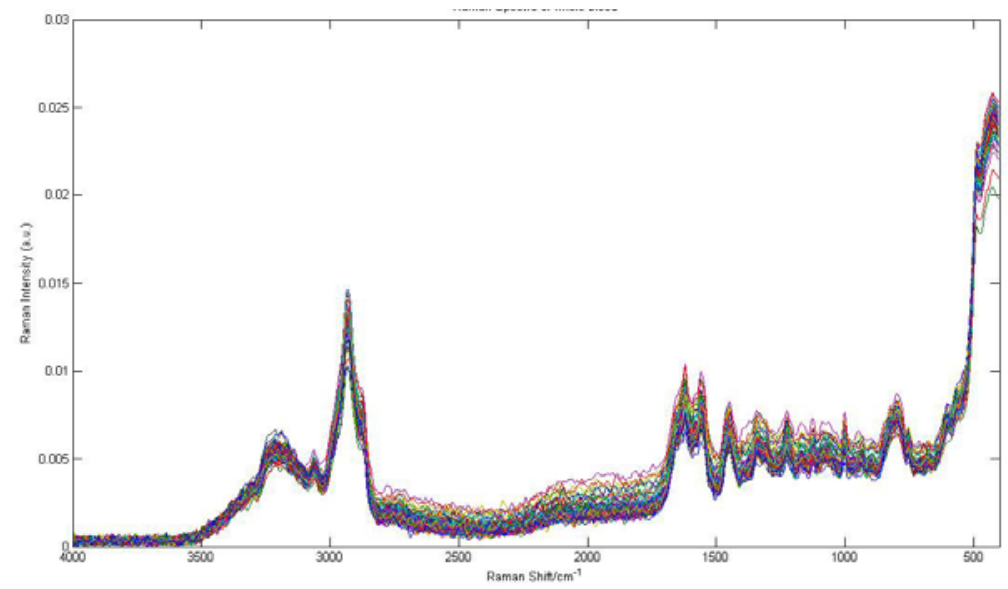

Figure 2. The Raman spectroscopy of whole blood.

Altogether 116 patients were available for measurement and testing after taking informed consent of which 101 patient data have been used for training the neural networks and testing has been carried out on remaining15 patients. The training for these neural networks using data of 80 patients has been done and then implemented on sample selection to minimize the errors. The training set was used to validate the predictive ability of the optimize neurons in ANN models as non-linear regression model. As shown in Fig.3, the R2 and RMSEP of validation set for ANN model are 0.97647 and $0.502 \mathrm{mmol} / \mathrm{l}$. the results show that the ANN model give satisfactory fitting results and predictive ability, and the tand $\mathrm{F}$ tests which were used to compare the predicted values to the reference values indicated that the accuracy. 

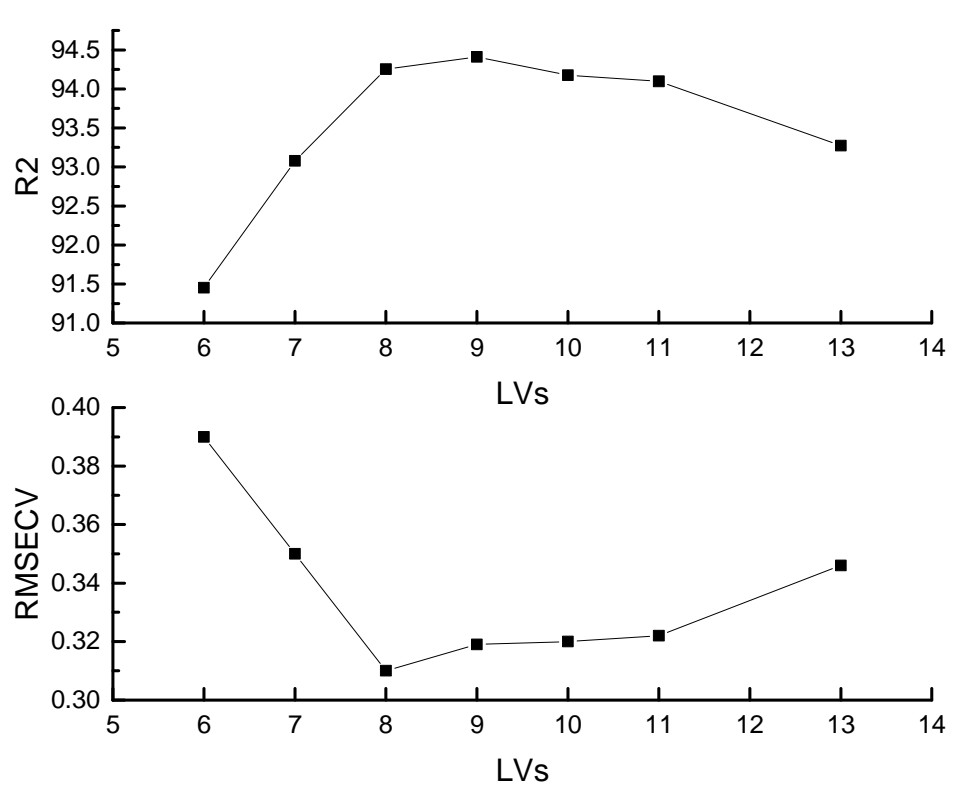

Figure 3 the dependency of RMSECV and R2 on the number of latent variable

\section{Discussion and Conclusions}

The current work focuses on estimation of blood glucose concentration based on Raman spectroscopy and signal processing thereafter using ANNs. The training and testing of neural networks has been carried out in MATLAB with real patient data. The correspondence between the estimated value by the neural network and the actual blood glucose concentration of the patient obtained by conventional pathological lab test was found. The number of neurons in the hidden layer of neural networks has been suitably increased to improve the accuracy in estimation of blood glucose. The results show that the RMSEP in estimating glucose has been found to be $0.502 \mathrm{mmol} / \mathrm{l}$. A correlations coefficient is 0.97647 . The good performance and extensive potential of ANNs is shown in measurement of blood glucose, and it will be carried out for human noninvasive in our future study.

Diabetes, which is caused by the disorder of blood glucose, has been one of the most important metabolic diseases worldwide. In order to avoid the diabetes-related complications, such as blindness and loss of limbs, the diet adjustment and insulin therapy are need to check glucose level at least four to five times a day. The aim of this paper was to investigate the potential of Fourier-Transform Raman spectroscopy as a diagnostic tool to monitor the blood glucose, triglycerides and total cholesterol levels. Raman spectroscopy were acquired from 116 individuals with diabetes patients, patients and healthy patients in order to gain an insight into the determination of biochemical changes for the diabetes diagnose. The human whole blood was examined at 1064nm excitation laser source. A new method with modified sample selection algorithm named (K-Means) KM is developed and applied to the Raman spectroscopy of whole blood. The algorithms have been successfully applied to improve the prediction accuracy. This method can detect the glucose concentration in the human whole blood without complicated sample preparation procedures. The experimental results show that the Raman spectroscopy technology has enormous clinical potential as a rapid diagnostic tool for diabetes diseases.

\section{Acknowledgements:}

This work was partly supported by the National Natural Science Foundation of China (11404054, 61601104), the Natural Science Foundation of Hebei Province (F2014501137, F2017501052), the Fundamental Research Funds for the Central Universities (N142304003). 


\section{References}

[1] Information on http://www.weld.labs.gov.cn

I.D. Federation, IDF diabetes atlas seventh edition, IDF Diabetes Atlas-Seventh Edition, (2015).

[2] A. Trabelsi, M. Boukadoum, M. Siaj, A preliminary investigation into the design of an implantable optical blood glucose sensor, American journal of biomedical engineering, 1(2011) 62-7.

[3] P. Lemler, W. Premasiri, A. DelMonaco, L. Ziegler, NIR Raman spectra of whole human blood: effects of laser-induced and in vitro hemoglobin denaturation, Analytical and bioanalytical chemistry, 406(2014) 193-200.

[4] S. Ramasahayam, S.H. Koppuravuri, L. Arora, S.R. Chowdhury, Noninvasive blood glucose sensing using near infra-red spectroscopy and artificial neural networks based on inverse delayed function model of neuron, J Med Syst, 39(2015) 166.

[5] S. Sharma, M. Goodarzi, H. Ramon, W. Saeys, Performance evaluation of preprocessing techniques utilizing expert information in multivariate calibration, Talanta, 121(2014) 105-12.

[6] M. Goodarzi, S. Sharma, H. Ramon, W. Saeys, Multivariate calibration of NIR spectroscopic sensors for continuous glucose monitoring, TrAC Trends in Analytical Chemistry, 67(2015) 147-58.

[7] Q. Wang, N. Zheng, Z. Li, Z. Ma, Ieee, Quantitative analysis of glucose in whole blood based on FT-Raman spectroscopy and back propagation artificial neural network, 2015 Ieee 7th International Conference on Awareness Science \& Technology2015, pp. 153-6.

[8] N.W. Tietz, Clinical guide to laboratory tests $4^{\text {th }}$ ed: WB Saunders Company; 1995.

[9] T. Weichselbaum, An accurate and rapid method for the determination of proteins in small amounts of blood serum and plasma, Am J Clin Pathol, 10(1946) 40-9.

[10] G.L. Myers, M.M. Kimberly, P.P. Waymack, S.J. Smith, G.R. Cooper, E.J. Sampson, A reference method laboratory network for cholesterol: a model for standardization and improvement of clinical laboratory measurements, Clin Chem, 46(2000) 1762-72. 